\title{
Collisional atomic mixing in polyatomic targets
}

\author{
J. A. Peinador, I. Abril, ${ }^{*}$ and J. J. Jiménez-Rodríguez \\ Departamento de Electricidad y Electrónica, Facultad de Ciencias Físicas, Universidad Complutense, \\ E-28040 Madrid, Spain \\ A. Gras-Marti \\ Department de Física Aplicada, Universitat d'Alacant, Apartat 99, E-03080 Alacant, Spain
}

(Received 11 October 1990)

\begin{abstract}
The balance equation derived by Sigmund, Oliva, and Falcone [Nucl. Instrum. Methods 194, 541 (1982)] for the evolution with ion fluence of the composition of irradiated polyatomic targets is generalized to include the effect of the implanted bombarding ions and the allowance for the target density to depend on depth. The model also includes atomic mixing, surface recession due to sputtering, and homogeneous target relaxation. As an additional feature in theoretical descriptions of collisional mixing, the need to introduce the effect of the surface in the relocation operator is stressed, and a model for the evaluation of this contribution is proposed. The relocation operators are evaluated in detail for power-law interatomic potentials. We give explicit expressions that are ready for use in numerical solutions of the coupled integro-differential equations that describe the concentration profiles. The potential of the formalism to treat a variety of topics in the interaction of energetic ions with matter is commented upon. In particular, we discuss the conditions for the balance equation to be linear, and results on the evolution with ion fluence of thin markers embedded in solids are compared with other theoretical predictions and with experimental data.
\end{abstract}

\section{INTRODUCTION}

The evaluation of the contribution of collisional mixing to the composition of irradiated targets, is still a felt need. While the number of experimental reports on mixing is large, ${ }^{1}$ theoretical predictions of the effects of mixing have been rather scarce in the last decade. A clear knowledge of the collisional contribution is important in order to fully appreciate the validity of alternative explanations of the results of mixing experiments. ${ }^{2}$

As in other issues within the field of atomic collisions in solids, computer simulations and analytical approaches are used to theoretically predict mixing profiles. Computer simulations, either based on Monte Carlo schemes or in molecular dynamics are scarce; they are expensive (in CPU time) and complicated, due to the high fluence of ions involved in mixing experiments. ${ }^{3,4}$ Analytical theories, based on transport equations of the Boltzmann type,$^{5-7}$ are not any simpler to deal with because of the nonlinear and coupled character of the processes involved, and also because of the difficulty in developing analytical results even for simplified models of collisional relocation events. Analytical theories usually treat two extreme limits concerning the target, the dilute limit ${ }^{6}$ or the nearly equal-mass limit. ${ }^{5}$ Diffusion approximations of transport equations for mixing have also been extensively investigated. ${ }^{8}$

Since the derivation ${ }^{9}$ in 1982 of a balance equation for the evolution with ion fluence of the composition of sputtered polyatomic targets, including atomic mixing, surface recession due to sputtering, and homogeneous target relaxation, little practical use has been made of such a powerful theoretical scheme. Only formal solutions of those equations were obtained for artificial collisional cross sections, ${ }^{9,10}$ or with numerous approximations. ${ }^{11}$ We have shown that the spatial dependence of the coefficients in the balance equation cannot be approximated as constants with any reasonable value. ${ }^{12}$ In this paper, the coefficients in the coupled, nonlinear, integrodifferential set of equations that describe target composition are evaluated for realistic cross sections for recoil and cascade-induced collisional-mixing mechanisms.

The effect of the bombarding ions on mixing has been treated ${ }^{13}$ neglecting the effect of target relaxation. Here, however, the model proposed ${ }^{9}$ is generalized to include the effect of the bombarding ions that are implanted in the solid. This adds another important nonlinear effect at high ion fluences.

Up to this point, the expanded formalism that we propose to investigate is simply an alternative, but equivalent approach to the one developed by Littmark and Hofer. ${ }^{5,14}$ There are, however, some differences in the explicit form of the evolution equation that each group of authors proposes ${ }^{9,5}$ and, furthermore, it is of interest to have access to independent calculations of such complicated effects as the ones mentioned in this paper. On top of this, our model explicitly incorporates the target surface into the atomic mixing process.

In our view, the need to introduce the effect of the surface barrier in the relocation function has to be stressed. The surface barrier is a well-established feature ${ }^{15}$ in atomic collision theory, affecting mainly the sputtering event. The surface barrier plays an important role in the relocation of atoms close to the surface and in the predictions of appropriate sputtering yields. But sputtering is only one of the possible outcomes of atomic relocation events close to the surface. A model for the evaluation of the surface effect is proposed in this paper, and specified for 
the most significant mixing processes. This discussion of the effect of the surface seems to be missing in previous theoretical descriptions of collisional mixing. ${ }^{9,5-7,14}$

The computer code TUI (target under irradiation) has been developed, based on the present formalism, and has been applied to treat the sputtering of adsorbed monolayers on a substrate, ${ }^{16}$ the evolution with ion fluence of thin markers in solids, ${ }^{17}$ the dynamic recoil mixing technique, ${ }^{18}$ the accumulation of implanted ions with fluence, the sputtering of isotopic targets, the characterization of depth profiling by sputtering, the preferential sputtering of compounds, and others. The detailed input used in the calculations reported ${ }^{16-18}$ will be given here.

In Sec. II the inclusion of implanted ions in the balance equation ${ }^{9}$ for the evolution with fluence of the composition of polyatomic targets is discussed. The relocation operators, for recoil-mixing (RM) and cascade-mixing (CM) mechanisms, with the incorporation of the surface effect, will be specified in Sec. III and, finally, in Sec. IV, this formalism will be applied to a particular case where the balance equation is linear.

\section{GENERAL FORMALISM}

Take a projectile of mass $M_{0}$, impinging up to a fluence $\phi$ (ions $/ \mathrm{cm}^{2}$ ) on a polyatomic solid with atomic masses $M_{i}$. At an ion fluence $\phi$, let $\alpha_{i}(x, \phi)$ be the volume atomic fraction of the projectiles implanted in the solid $(i=0)$, or of the target species $(i=1,2, \ldots, n)$, at a depth $x$ from the bombarded surface located at $x=0$. Volume atomic fractions $\alpha_{i}(x, \phi)$ are given by $\alpha_{i}=N_{i} \Omega_{i}$, in terms of the effective atomic volume $\Omega_{i}$, and the atomic density $N_{i}(x, \phi)$ of the species $i$.

The balance equations for the evolution with ion fluence of the volume atomic fractions of all the atomic species that compose the target $(i=0,1, \ldots, n)$, are obtained after a slight generalization of the formalism developed by Sigmund et al.,

$$
\begin{aligned}
\frac{\partial \alpha_{i}(x, \phi)}{\partial \phi}= & L_{i} \alpha_{i}(x, \phi)-\frac{\partial}{\partial x}\left[\alpha_{i}(x, \phi) \int_{0}^{x} d x^{\prime} h\left(x^{\prime}, \phi\right)\right] \\
& +\delta_{i 0} \Omega_{0} \mathcal{F}_{0}^{R}(x, E, \eta, \phi) .
\end{aligned}
$$

The relocation operator $L_{i}$ is related to the relocation cross sections, so that $L_{i} N_{i}(x, \phi)$ expresses the net mean number of $i$ atoms relocated to a depth $x$, per unit fluence. $h(x, \phi)$ in Eq. (1) is the relaxation function that prevents the occurrence of over- or underaccumulation of atoms in the solid by allowing the total density to vary according with the effective volume of the atoms that are filling a given volume of the solid. The last term on the right-hand side of Eq. (1), not included in the equation derived by Sigmund et al., ${ }^{9}$ accounts for the accumulation of projectiles in the target. $\mathcal{F}_{0}^{R}(x, E, \eta, \phi)$ is the range distribution ${ }^{19}$ of the projectile in the polyatomic target, for an initial energy $E$ and a direction $\theta(\eta=\cos \theta)$ with respect to the surface normal, after a fluence $\phi$.

In the derivation of Eq. (1), a homogeneous target relaxation is assumed according with the following stability criterion:

$$
\sum_{i=0}^{n} \alpha_{i}(x, \phi)=\sum_{i=0}^{n} \Omega_{i} N_{i}(x, \phi)=1
$$

for all $x$ and $\phi$. Then the relaxation function $h(x, \phi)$ will be given by

$$
h(x, \phi)=\sum_{i=0}^{n} L_{i} \alpha_{i}(x, \phi)+\Omega_{0} \mathcal{F}_{0}^{R}(x, E, \eta, \phi) .
$$

The stability condition in Eq. (2) may be made more general and one obtains the relaxation function given in Appendix A. In general, the range distribution $\mathscr{F}_{0}^{R}(x, E, \eta, \phi)$, the relocation operators $L_{i}$, and the relaxation function $h(x, \phi)$ will all depend on the volume atomic fractions $\alpha_{i}(x, \phi), i=0,1, \ldots, n$. We have expressed this dependence by $\phi$ in order to simplify the notation.

Now we discuss the relocation operator. For a realistic description of semi-infinite media, we explicitly include the effect of the surface and its concomitant surface barrier through the relocation operator $L_{i}$. One has to deal with those atoms that reach the surface with insufficient energy to overcome the surface barrier. We introduce a surface backscattering function $W_{i}\left(x^{\prime}, x, \phi\right)$, such that, at fluence $\phi$,

$$
N_{i}\left(x^{\prime}, \phi\right) W_{i}\left(x^{\prime}, x, \phi\right) d x^{\prime} d x
$$

is the average number of $i$ atoms that, per incoming ion, having been displaced from $\left(x^{\prime}, d x^{\prime}\right)$, reach the surface without enough energy to overcome the surface barrier; these particles are backscattered at the surface, and slow down in the target to stop at a depth $(x, d x)$. It is not obvious that this effect has been taken into account in previous treatments. ${ }^{3,5,14}$ One should note that the need to account for this possibility arose quite naturally when trying to keep track of all atoms (mass conservation) relocated after a given fluence, and in order to predict reasonable sputtering yields.

The relocation operator $L_{i}$ is therefore an extension of the one defined in the literature ${ }^{9,6,7}$ in that it takes into account the surface effect

$$
\begin{aligned}
L_{i} \alpha_{i}(x, \phi)= & \int_{-\infty}^{x} d \sigma_{i}(x-z, z, \phi) \alpha_{i}(x-z, \phi) \\
& +\int_{0}^{+\infty} d x^{\prime} W_{i}\left(x^{\prime}, x, \phi\right) \alpha_{i}\left(x^{\prime}, \phi\right) \\
& -\int_{-\infty}^{+\infty} d \sigma_{i}(x, z, \phi) \alpha_{i}(x, \phi) .
\end{aligned}
$$

The second term on the right-hand side accounts for the surface barrier. As in Refs. 6 and 7, the relocation cross sections are defined without considering the surface barrier, which is included through the surface backscattering function $W_{i}\left(x^{\prime}, x, \phi\right)$. In Eq. (4), $d \sigma_{i}(x, z, \phi)$ is the differential relocation cross section for an $i$ atom, ${ }^{6}$ so that $d \phi d \sigma_{i}(x, z, \phi)$ is the probability that an $i$ atom is relocated from the layer $(x, d x)$ to the layer $(x+z, d z)$ after the fluence increment $d \phi$. The dependence of the cross section on all $\alpha_{i}$ is symbolically contained in $\phi$.

The partial sputtering yield of the $i$ atoms, $Y_{i}(\phi)$, is then given by 


$$
\begin{aligned}
Y_{i}(\phi)= & \int_{0}^{+\infty} d x N_{i}(x, \phi) \int_{-\infty}^{-x} d \sigma_{i}(x, z, \phi) \\
& -\int_{0}^{+\infty} d x \int_{0}^{+\infty} d x^{\prime} W_{i}\left(x^{\prime}, x, \phi\right) N_{i}\left(x^{\prime}, \phi\right),
\end{aligned}
$$

where the first term on the right-hand side represents the sputtering yield without taking into account any surfacebarrier effect; the second term counts all the relocated $i$ atoms that are not able to overcome the surface barrier. We can write Eq. (5) as ${ }^{9}$

$$
Y_{i}(\phi)=\int_{0}^{+\infty} d x N_{i}(x, \phi) \sigma_{i}^{s}(x, \phi),
$$

with a sputtering cross section, $\sigma_{i}^{s}(x, \phi)$, given by

$$
\sigma_{i}^{s}(x, \phi)=\int_{-\infty}^{-x} d \sigma_{i}^{\prime}(x, z, \phi) .
$$

$d \sigma_{i}^{\prime}(x, z, \phi)$ is now the relocation cross section calculated accounting for the surface barrier. Comparing Eqs. (5), (6), and (7) one gets

$$
\sigma_{i}^{s}(x, \phi)=\int_{-\infty}^{-x} d \sigma_{i}(x, z, \phi)-\sigma_{i}^{r}(x, \phi),
$$

where, in an analogous manner to the definition of the sputtering cross section, we define a surface backscattering cross section, $\sigma_{i}^{r}(x, \phi)$, as

$$
\sigma_{i}^{r}(x, \phi)=\int_{0}^{+\infty} d x^{\prime} W_{i}\left(x, x^{\prime}, \phi\right) .
$$

$N_{i}(x, \phi) d x \sigma_{i}^{r}(x, \phi)$ is the mean number of $i$ atoms, initially located at depth $(x, d x)$, which, being relocated, reach the surface but do not have enough energy to overcome it. Equation (8) relates the relocation, sputtering, and surface-backscattering cross sections.

\section{RELOCATION OPERATORS: CROSS SECTIONS}

In order to solve the balance Eq. (1), the relocation operators $L_{i}$, in Eq. (4), for each collisional mixing mechanism need to be specified. We shall generalize the relocation cross sections ${ }^{6}$ for isotropic-cascade and recoilmixing processes, and shall model the surfacebackscattering function to include the surface effect in the relocation operator.

\section{A. Isotropic cascade mixing}

Isotropic cascade mixing refers to the relocation of atoms in the collisional cascades developed in the target by energetic ions and energized recoils. In a polyatomic target, the relocation cross section of $i$ atoms, due to all $k$ atoms, is

$$
d \sigma_{i}(x, z, \phi)=\sum_{k=0}^{n} d \sigma_{k i}(x, z, \phi) .
$$

We introduce ${ }^{19}$ the functions $H_{k}=\Sigma_{j} H_{j k}$ defined as follows: Per particle of species $j$, with energy $E$ and direction $\mathbf{\Omega}$,

$$
H_{j k}\left(x, E, \mathbf{\Omega}, E_{0}, \mathbf{\Omega}_{0}, \phi\right) d E_{0} d^{2} \Omega_{0}
$$

is the average number of $k$ atoms, at fluence $\phi$, that cross the plane $x$, with energy in the interval $\left(E_{0}, d E_{0}\right)$ and direction within the solid angle $\left(\Omega_{0}, d^{2} \Omega_{0}\right)$. In the limit $E_{0} \ll E$, it has been shown ${ }^{20}$ that the recoil distributions $H_{j k}$ are isotropic. Then the relocation cross section for this isotropic mechanism will be given by ${ }^{6}$

$$
d \sigma_{i}^{\mathrm{CM}}(x, z, \phi)=d z \sum_{k=0}^{n} \int d E_{0} \int d^{2} \Omega_{0} H_{k}\left(x, E, \mathbf{\Omega}, E_{0}, \mathbf{\Omega}_{0}, \phi\right) \frac{1}{\cos \theta_{0}} \int d \sigma_{k i}\left(E_{0}, \mathbf{\Omega}_{0}, T, \mathbf{\Omega}^{\prime}\right) \mathcal{F}_{i}^{R}\left(z, T-E_{d i}, \mathbf{\Omega}^{\prime}, \phi\right),
$$

where $d \sigma_{k i}\left(E_{0}, \Omega_{0}, T, \Omega^{\prime}\right)$ is the differential energy-transfer cross section in a collision between the atoms $k$ and $i ; T$ and $\mathbf{\Omega}^{\prime}$ are, respectively, the energy and the direction of the recoil $i$ atom and $E_{d i}$ is a threshold displacement energy for $i$ type atoms. The relocation cross section for the cascade-mixing process is given by expression (11), without considering the effect of the surface barrier. This latter effect will be taken into account through the surface-backscattering function as defined in Eqs. (4) and (5).

In order to determine the surface-backscattering function $W_{i}\left(x^{\prime}, x, \phi\right)$, we shall first assume that the surface barrier is planar and of height $U$; thus, those atoms reaching the surface with energy $E_{s}$ and direction $\theta_{s}$, such that

$$
E_{s} \leq U / \cos ^{2} \theta_{s}=U / \eta_{s}^{2}
$$

shall not be able to overcome the barrier. Second, we assume that these atoms that cannot overcome the surface barrier are specularly backscattered without suffering any energy loss at the surface and are consequently slowed down in the solid. With these assumptions, the surface-backscattering function $W_{i}\left(x^{\prime}, x, \phi\right)$ of the $i$ species is given by

$$
W_{i}\left(x^{\prime}, x, \phi\right)=\frac{1}{N_{i}\left(x^{\prime}, \phi\right)} \int d^{2} \Omega_{s} \int_{E_{s} \leq U / \eta_{s}^{2}} d E_{s} J_{i}\left(E_{s}, \mathbf{\Omega}_{s}, x^{\prime}, \phi\right) \delta\left[x-R_{i}\left(E_{s}, \phi\right) \eta_{s}\right],
$$

where $R_{i}\left(E_{s}, \phi\right)$ is the path length traveled by an $i$ atom of energy $E_{s}$, at fluence $\phi . J_{i}\left(E_{s}, \Omega_{s}, x, \phi\right) d E_{s} d^{2} \Omega_{s} d x$ is ${ }^{21}$ the average number of $i$ atoms recoiling in the layer $(x, d x)$ due to an elastic collision cascade, and reaching the surface $(x=0)$ with energy $\left(E_{s}, d E_{s}\right)$ and direction $\left(\boldsymbol{\Omega}_{s}, d^{2} \Omega_{s}\right)$. This function is given by

$$
\begin{aligned}
J_{i}\left(E_{s}, \mathbf{\Omega}_{s}, x, \phi\right)=\sum_{k=0}^{n} \int d E_{0} \int & d^{2} \Omega_{0} H_{k}\left(x, E, \mathbf{\Omega}, E_{0}, \mathbf{\Omega}_{0}, \phi\right) \frac{N_{i}(x, \phi)}{\cos \theta_{0}} \\
& \times \int d \sigma_{k i}\left(E_{0}, \mathbf{\Omega}_{0}, T, \mathbf{\Omega}^{\prime}\right) \int d^{2} \mathbf{\Omega}^{\prime} \delta\left(\mathbf{\Omega}^{\prime}-\mathbf{\Omega}_{s}\right) \delta\left(E_{s}-f_{i}\left(x, T-E_{d i}, \mathbf{\Omega}^{\prime}, \phi\right)\right),
\end{aligned}
$$


where the functional dependence $E_{s}=f_{i}\left(x, T, \mathbf{\Omega}^{\prime}, \phi\right)$ expresses the energy $E_{s}$ with which an $i$ atom reaches the surface, after having left the depth $x$ with energy $T$ and direction $\Omega^{\prime}$. We use the continuous slowing-down approximation along straight lines for the slowing down of the $i$ atom in the target. This approximation should be taken with some precaution for the slowing down of light species in polyatomic targets. However, in studies of sputtering there is some evidence that the slowing-down approximation may predict well the depth of origin of the sputtered particles, ${ }^{22}$ due to compensation of scattering processes.

Introducing expressions (11) and (12) into Eq. (4), we can calculate the relocation operators $L_{i}$ for the isotropic cascade-mixing mechanism with the explicit inclusion of the surface effect. At this point, one may reconsider the need to account for the surface-barrier potential. Alternatively, observed sputtering yields could be predicted by a proper choice of the threshold displacement energy $E_{d i}$. Beside the fact mentioned above that the use of a surface barrier is very widely accepted, ${ }^{15}$ the neglect of the surface barrier requires very large values of $E_{d i}$ in order to fit the sputtering yields. For instance, in the case which will be studied later of $300 \mathrm{keV}$ xenon bombarding silicon, an $E_{d}=55 \mathrm{eV}$ is required to obtain the same sputtering yield as for a binding surface energy $U=4.63$ $\mathrm{eV}$ and $E_{d}=15 \mathrm{eV}$. On the other hand, values in the literature ${ }^{23,24}$ are in the range $E_{d}: 10-20 \mathrm{eV}$ for $\mathrm{Si}$.

\section{B. Recoil mixing}

Recoil mixing refers to the relocation of target atoms in direct collisions with the incident ions. Therefore, for this mechanism there is only a contribution from the projectile.

The relocation cross section for an $i$ atom, at depth $x$, due to a direct collision with a projectile of energy $E(x, \phi)$, is given by ${ }^{6}$

$$
\begin{aligned}
& d \sigma_{i}^{R M}(x, z, \phi)=d z \int d \sigma_{0 i}[E(x, \phi), T] \\
& \times \delta\left[z-R_{i}\left(T-E_{d i}, \phi\right) \cos \psi_{i}\right] \\
& \text { for } 0 \leq z \leq z_{i}^{\max }(x),
\end{aligned}
$$

where the projectile is assumed to hit the solid in the direction of the surface normal. $\psi_{i}$ is the scattering angle for the recoiling $i$ atom. The $\delta$ function comes from the continuous slowing-down approximation along straight lines, for the slowing down of the recoiling particles in the solid. This model may be a poor approximation for light atomic species.

In this recoil-mixing model via direct collision of a projectile with an $i$ target atom $(i=0,1, \ldots, n)$, it is not possible for the target atom to be scattered towards the surface, at least for normal incidence of the projectile. Therefore, in comparison with Eq. (4), the relocation operator $L_{i}$ for this mechanism

$$
\begin{aligned}
L_{i} \alpha_{i}(x, \phi)= & \int_{0}^{x} d \sigma_{i}(x-z, z, \phi) \alpha_{i}(x-z, \phi) \\
& -\int_{0}^{+\infty} d \sigma_{i}(x, z, \phi) \alpha_{i}(x, \phi)
\end{aligned}
$$

contains no surface-barrier term. Furthermore, the lower limit of both integrals in Eq. (15) are now set equal to zero because this is a directional mechanism moving particles inside the target.

Recent computer-simulation studies ${ }^{25}$ of the relocation cross section have shown that this restriction to forward relocation is not bad in view of the relative magnitude of forward and backward contribution to the relocation cross section.

\section{APPLICATION: LINEAR CASES}

There are some simple applications of this formalism which are of practical interest, namely, the case of a target whose species have similar masses, ${ }^{5}$ and the case of an impurity diluted into a matrix, ${ }^{6}$ when these systems are bombarded with not too high ion fluences. In these limits the balance equation (1) is linear, the target can be treated like monoatomic for the slowing down of the particles, and the relocation operator will not depend on the atomic concentrations in the target. For high-energy ions, one may also neglect the accumulation of projectiles in the solid. Applications of the present formalism to such systems have been briefly reported. ${ }^{16-18}$ The input used in those calculations will be specified here.

For a diluted impurity $M_{1}$ in a matrix $M_{2}$, where $\alpha_{1}<<\alpha_{2}, \alpha_{2} \simeq 1$ is already a solution of Eq. (1) (monoatomic target). The evolution equation for the impurity atoms $\alpha_{1}$ is linear

$$
\frac{\partial \alpha_{1}}{\partial \phi}=L_{1} \alpha_{1}-\frac{\partial}{\partial x}\left[\alpha_{1} \int_{0}^{x} d x^{\prime} L_{2}^{\prime}\right],
$$

since the relaxation function [see Eq. (3)] can be written as

$$
h\left(x^{\prime}\right)=L_{1}\left(x^{\prime}\right) \alpha_{1}+L_{2}\left(x^{\prime}\right) \alpha_{2} \simeq L_{2}\left(x^{\prime}\right) \equiv L_{2}^{\prime} .
$$

For the nearly equal-mass target limit $M_{1} \simeq M_{2}$, the relocation operators also coincide $L_{1} \simeq L_{2}$, and Eq. (16) still holds for $\alpha_{1}$, while $\alpha_{2}=1-\alpha_{1}$. The relaxation operator will be the same as that for a diluted impurity in a matrix,

$$
h\left(x^{\prime}\right)=L_{1}\left(x^{\prime}\right) \alpha_{1}+L_{2}\left(x^{\prime}\right)\left(1-\alpha_{1}\right) \simeq L_{2}\left(x^{\prime}\right) \equiv L_{2}^{\prime} .
$$

In order to solve the balance equation (16) we need to specify the relocation operator (4) for isotropic-cascadeand recoil-mixing mechanisms, and the relocation cross sections and the surface-backscattering function need to be evaluated. For the case of a diluted impurity in a matrix, we calculate the relocation cross section of $i$ atoms $(i=1,2)$, due to elastic collision cascades developed by matrix atoms only from Eq. (11). Since we are ignoring the effects due to the projectile, we substitute in Eq. (10),

$$
d \sigma_{i}(x, z) \cong d \sigma_{2 i}(x, z)
$$

and in Eq. (11), $H_{k} \cong H_{2 k}$. Using in Eq. (11) the wellknown expressions of $H_{2}\left(x, E, \Omega, E_{0}, \Omega_{0}\right)$ (Ref. 19) and the power-law energy-transfer cross section ${ }^{26,27}$ $d \sigma_{2 i}\left(E_{0}, \mathbf{\Omega}_{0}, T, \mathbf{\Omega}^{\prime}\right)$, we can find the relocation cross section. We generalize the results ${ }^{6}$ for different exponents 
$m_{i j}$ in the power-law interatomic potential and also consider a threshold displacement energy $E_{d i}$ in order to avoid the singularity at $z=0$. We obtain

$$
\begin{aligned}
d \sigma_{2 i}^{\mathrm{CM}}(x, z)= & d z \frac{\Gamma \mathcal{F}^{D}(x) C_{2 i}\left(1-m_{22}\right) \gamma_{2 i}^{1-2 m_{22}+m_{2 i}}}{2 N_{2} C_{22}\left(1-2 m_{22}+m_{2 i}\right) A_{i}} \\
& \times \int_{T_{0}}^{\infty} d T \frac{T^{-p_{i}}}{\left(T-E_{d i}\right)^{2 m_{i 2}}},
\end{aligned}
$$

where $p_{i}=2-2 m_{22}+2 m_{2 i}$ and

$$
T_{0}=E_{d i}+\left(|z| / A_{i}\right)^{1 / 2 m_{i 2}} .
$$

$\Gamma$ is a well-known ${ }^{19}$ parameter depending on $m_{i j}, \mathcal{F}^{D}(x)$ is the deposited energy distribution ${ }^{27}$ of the projectile into the matrix, and $A_{i}$ is a parameter in the range of the recoil atom $i$ in the matrix, i.e., $R_{i}(E)=A_{i} E^{2 m_{i 2}}$.

There is a simple analytical solution for the relocation cross section when $m_{i 2}=\frac{1}{4}$ and $m_{22}=m_{2 i}$,

$$
\begin{aligned}
d \sigma_{2 i}^{\mathrm{CM}}(x, z)= & d z \frac{\Gamma \mathcal{F}^{D}(x) C_{2 i} \gamma_{2 i}^{1-m_{22}}}{2 N_{2} C_{22} A_{i} E_{d i}} \\
\times & {\left[\frac{\pi}{2 E_{d i}^{1 / 2}}-\frac{|z|}{A_{i}\left[E_{d i}+\left(|z| / A_{i}\right)^{2}\right]}\right.} \\
& \left.-\frac{1}{E_{d i}^{1 / 2}} \tan ^{-1}\left[\frac{|z|}{A_{i} E_{d i}^{1 / 2}}\right]\right]
\end{aligned}
$$

In general, for different values of $m_{i j}$ it is still possible to get analytical expressions by expanding the $T$ integral in Eq. (17) as a series ${ }^{28}$ (for details see Appendix B).

It is interesting to note the behavior of the relocation cross section with the threshold energy parameter $E_{d i}$. For large $z$ values (large relocation distances) we reproduce the results of Sigmund and Gras-Marti, ${ }^{6}$ where this threshold was not included, i.e., the cross sections are not sensitive to the values of $E_{d i}$ chosen and the slopes are identical because they only depend on the $m$ values chosen. In the range of small $z$ values, the relocation cross section is quite sensitive to the specified $E_{d i}$ and the curves start to bend to a constant value for $z=0$ around a value of $z$ corresponding to the path length of a recoil with energy $E_{d i}$. The probability for an atom to be relocated a small distance $z$ is larger the smaller the displacement energy $E_{d i}$ because less energy needs to be transferred to move atoms, and the energy-transfer cross section is larger. For $E_{d i}=0$, the relocation cross section diverges at $z=0$. The $m$ dependence of the cascademixing relocation cross section is the following: for large $z$ the slopes are softer the higher the $m$ value. For small $z$, the larger the $m$ values, the shorter is the path length corresponding to $E_{d i}$ so that one has higher values for the isotropic relocation cross section.

Now, in order to find the relocation operator we need to specify the surface-backscattering function, Eq. (12). We obtain

$$
W_{i}\left(x^{\prime}, x\right)=\frac{\Gamma \mathcal{F}^{D}\left(x^{\prime}\right) C_{2 i}\left(1-m_{22}\right) \gamma_{2 i}^{1-2 m_{22}+m_{2 i}}}{4 N_{2} C_{22} A_{i} m_{i 2}\left(1-2 m_{22}+m_{2 i}\right)}\left(1+\frac{x^{\prime}}{x}\right]^{\left(1-2 m_{i 2}\right) / 2 m_{i 2}} \int_{\eta_{0}}^{1} \frac{d \eta_{s}}{\eta_{s}} \frac{\left(x / A_{i} \eta_{s}\right)^{\left(1-2 m_{i 2}\right) / 2 m_{i 2}}}{\left\{E_{d i}+\left[\left(x+x^{\prime}\right) / A_{i} \eta_{s}\right]^{\left.1 / 2 m_{i 2}\right\}^{p_{i}}}\right.}
$$

with

$$
\eta_{0}=\left(\frac{x}{A_{i} U^{2 m_{i 2}}}\right)^{1 /\left(1-4 m_{i 2}\right)}
$$

As for the cascade relocation cross section, we obtain a simple analytical expression from Eq. (19) when $m_{i 2}=\frac{1}{4}$ and $m_{22}=m_{2 i}$,

$$
\begin{aligned}
& W_{i}\left(x^{\prime}, x\right)= \frac{\Gamma \mathcal{F}^{D}\left(x^{\prime}\right) C_{2 i} \gamma_{2 i}^{1-m_{22}}}{2 N_{2} C_{22} A_{i}} E_{d i}^{-3 / 2} \\
& \times\left[\frac{\pi}{2}-\tan ^{-1}\left(\frac{x+x^{\prime}}{A_{i} E_{d i}^{1 / 2}}\right]\right. \\
&\left.-\frac{A_{i} E_{d i}^{1 / 2}\left(x+x^{\prime}\right)}{A_{i}^{2} E_{d i}+\left(x+x^{\prime}\right)^{2}}\right] .
\end{aligned}
$$

For different $m_{i j}$ we can also find an analytical result by expanding the integral in a series ${ }^{28}$ (for details see Appen$\operatorname{dix}$ B).

Figure 1 shows the depth distribution of the atoms reflected by the surface barrier in the target, i.e., $\int d x^{\prime} W_{i}\left(x^{\prime}, x\right)$. The system is $300-\mathrm{keV} \mathrm{Xe}$ ions incident

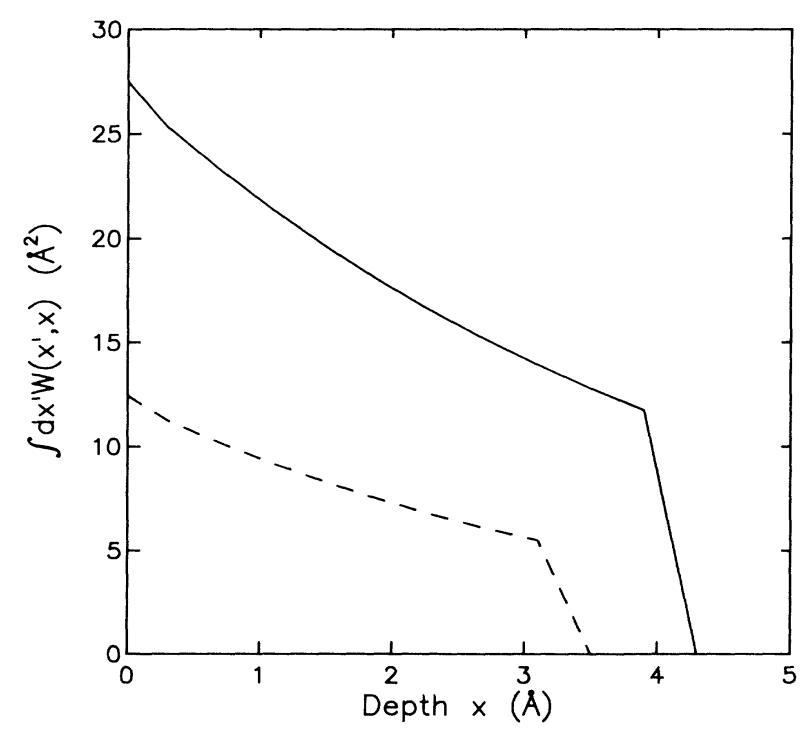

FIG. 1. Depth distribution of atoms reflected at the inner surface of the solid, for $300-\mathrm{keV} \mathrm{Xe} \rightarrow \mathrm{Si}(\mathrm{Pt})$. For $\mathrm{Si}(-)$ and $\mathrm{Pt}(---)$. 


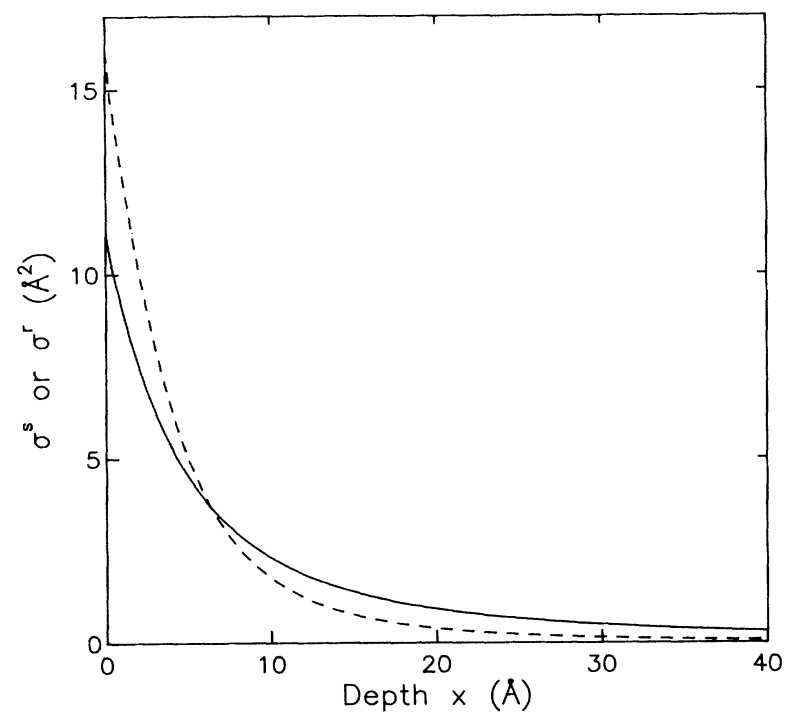

FIG. 2. Sputter (-) and surface reflection $(---)$ cross sections vs depth of silicon. For $300-\mathrm{keV} \mathrm{Xe} \rightarrow \mathrm{Si}(\mathrm{Pt})$.

on $\mathrm{Pt}$ diluted in $\mathrm{Si}$, a system that has been widely studied theoretically, ${ }^{6}$ computationally, ${ }^{29,30}$ and experimentally. ${ }^{31-33}$ We use $m=0.25, E_{d i}=15 \mathrm{eV}$, and $U=4.63 \mathrm{eV}$. No reflected atom can go further than the path length corresponding to the surface-barrier energy $U$ (a few $\AA$ ). It is precisely for values close to this path length where the depth distribution drops rapidly to zero. Also, the heavier Pt atoms have smaller recoil energies and ranges. Physically, when the target concentration is relaxed after each fluence increment, this model of the surfacebackscattering function gives very similar results to a model where those particles that cannot overcome the barrier are left at the surface. However, the proposed model is more convenient computationally.

Figure 2 shows the sputtering and reflection cross sections versus depth for silicon, as obtained from Eqs. (8) and (9), with the input specified above. The system and the parameters are the same as in Fig. 1. The sputtering yield obtained using Eq.(6) is 4.5 for silicon, a result that agrees quite well with experimental data. ${ }^{34}$ One observes in Fig. 2 that $\sigma^{s}>\sigma^{r}$ in the tail of the distribution, but the reverse holds near the surface. The behavior can be understood in terms of the energy and direction of the recoil atoms. It is well known that those atoms that reach the surface are more focused toward the surface normal the larger the depth where they come from. Those atoms moving at glancing directions contribute mainly to the reflection cross section $\left(E_{s} \leq U / \eta_{s}^{2}\right)$, whereas the contribution to the sputter cross section is mainly from atoms whose directions are close to the surface normal $\left(E_{s} \geq U / \eta_{s}^{2}\right)$.

Close to the surface, $\sigma^{r}>\sigma^{s}$ in Fig. 2. In the nearsurface region, atoms with any direction are able to reach the surface even if they have low energy. However, the opposite situation $\sigma^{r}<\sigma^{s}$ may occur if the height of the surface barrier is substantially reduced. At large $x$, however, $\sigma^{r}>\sigma^{s}$, independent of the height of the surface barrier because of the focusing effect.

The cascade-mixing contribution to the relocation operator $L_{i}$, given by Eqs. (4), (17), and (19), shows a depletion $^{12}$ around the maximum of the deposited energy distribution $\mathcal{F}^{D}$ and an enrichment region deeper inside. If the surface-barrier effect is included, a substantial enrichment is obtained in a very small region close to the surface (see Fig. 1); conversely, if the surface barrier is removed, then a pronounced depletion due to sputtering is found. ${ }^{12}$

Now we obtain the relocation cross section for the recoil-mixing mechanism from Eq. (14). We have calculated the scattering angle of the recoil particles $i$, with or without subtracting $E_{d i}$ to the transferred energy, and the differences in the calculated relocation cross section are negligible. Therefore, we use the scattering angle

$$
\cos \psi_{i}=\left[\left(T-E_{d i}\right) / \gamma_{0 i} E(x, \phi)\right]^{1 / 2}
$$

because, in this case, we obtain an analytical expression for the cross section in Eq. (14):

$$
\begin{aligned}
d \sigma_{i}^{R M}(x, z)= & d z \frac{C_{0 i}[E(x)]^{-m_{0 i}}}{1 / 2+2 m_{i 2}}\left[E_{d i}+\left[z \frac{\sqrt{\gamma_{0 i} E(x)}}{A_{i}}\right]^{1 /\left(1 / 2+2 m_{i 2}\right)}\right]^{-1-m_{0 i}} \\
& \times\left[\frac{\sqrt{\gamma_{0 i} E(x)}}{A_{i}}\right]^{1 /\left(1 / 2+2 m_{i 2}\right)} z^{1 /\left(1 / 2+2 m_{i 2}\right)-1} \quad\left[0 \leq z \leq z_{i}^{\max }(x)\right] .
\end{aligned}
$$

The relocation distance $z$ ranges from zero to

$z_{i}^{\max }(x)=A_{i}\left[\gamma_{0 i} E(x)-E_{d i}\right]^{2 m_{i 2}}\left(\frac{\gamma_{0 i} E(x)-E_{d i}}{\gamma_{0 i} E(x)}\right)^{1 / 2}$,

i.e., the maximum path length of a recoil $i$ at depth $x$. At $z=0$ the cross section in Eq. (21a) only vanishes for $m_{i 2}<\frac{1}{4}$, becoming divergent again for $m_{i 2}>\frac{1}{4}$.

Figure 3 shows the evolution, with ion fluence, of the concentration profiles of a platinum marker in a silicon matrix, bombarded with $300-\mathrm{keV} \mathrm{Xe}$. The $\mathrm{Pt}$ marker is $10 \AA$ wide, and initially located at $600 \AA$. The parameters $E_{d i}$ and $U$ are the same as in Fig. 1 but now $m=0.25$ and $\frac{1}{3}$ for the cascade and recoil mechanisms, respectively. The marker profiles broaden and shift towards the surface as the fluence increases. The surface effect is not relevant in terms of the distortion of such a deep marker, converse to observations made for shallow markers. ${ }^{17}$ The main influence of the surface barrier, in this particular case, is to accurately predict the sputtering 


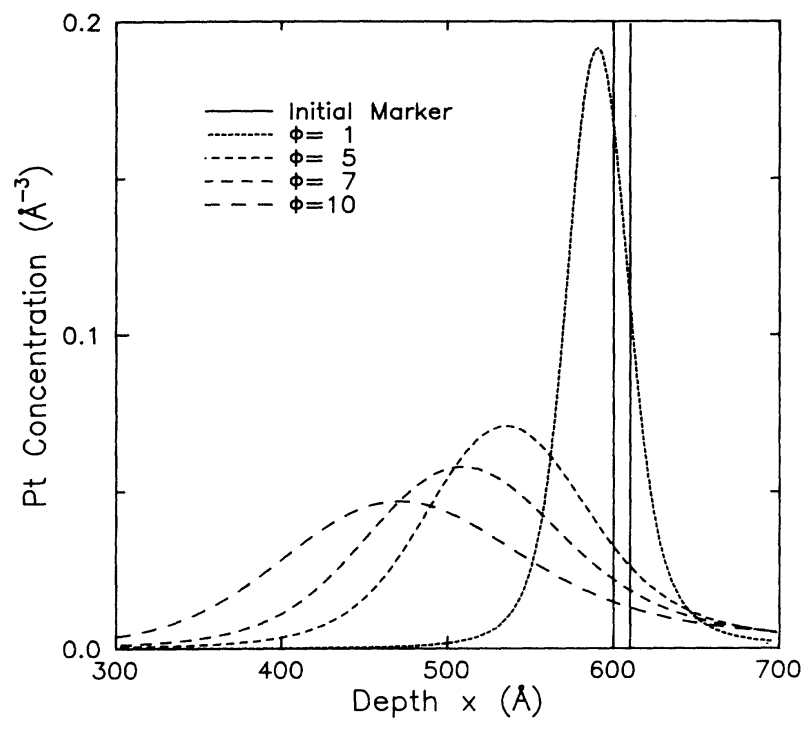

FIG. 3. Depth distribution of a Pt marker concentration for various fluences $\phi$ (in $10^{15}$ atoms $\mathrm{cm}^{-2}$ ), for $300-\mathrm{keV} \mathrm{Xe} \rightarrow$ $\mathrm{Si} / \mathrm{Pt} / \mathrm{Si}$.

yield so that the recession speed of the surface or, equivalently, the depth scale in Fig. 3, can be used for comparison with experimental profiles. The evolution of the $\mathrm{Pt}$ profile is very similar if we change the initial depth of the marker from 600 to $500 \AA$, as has been observed experimentally, ${ }^{35}$ due to the fact that $\mathcal{F}^{D}(x)$ does not vary much between those depths.

Figure 4 shows the broadening of a Pt marker as a function of ion fluence $\phi$. The system and parameters are the same as in Fig. 3. Figure 4(a) shows the mean standard deviation of the platinum marker. Experimental results $^{31}$ correspond to a 5 - $\AA$ width Pt marker, $500 \AA$ deep. We have found ${ }^{36} \sigma^{2}$ not to be sensitive when marker depth ranges between 400 and $800 \AA$, as has also been corroborated experimentally. ${ }^{35}$ Agreement between experiment and theory is quite reasonable, up to a fluence of about $10^{16} \mathrm{~cm}^{-2}$. At higher fluences the discrepancies may be due to the role played by other mechanisms, such as radiation enhanced diffusion, whose importance is expected to increase as the material is increasingly damaged.

Figure 4(b) shows the same kind of results but in terms of the half width at half maximum (HWHM). In this figure, we have also plotted $2(\ln 2) \sigma^{2}$ in order to indicate to what extent do the profiles shown in Fig. 3 depart from Gaussians ( $\sigma^{2}$ is the straggling in the profile). Theoretical results obtained by Monte Carlo simulation ${ }^{30}$ agree quite well with our predictions, keeping in mind that Monte Carlo calculations were carried out with a cutoff energy of $4 \mathrm{eV}$. Values of 8 and $25 \mathrm{eV}$, for the cutoff energy, reduce the magnitude of the square of the HWHM by, respectively, 20 and $50 \%$ approximately. It is interesting to remark that experimental values agree better with $2(\ln 2) \sigma^{2}$ than with the square of the HWHM, while they

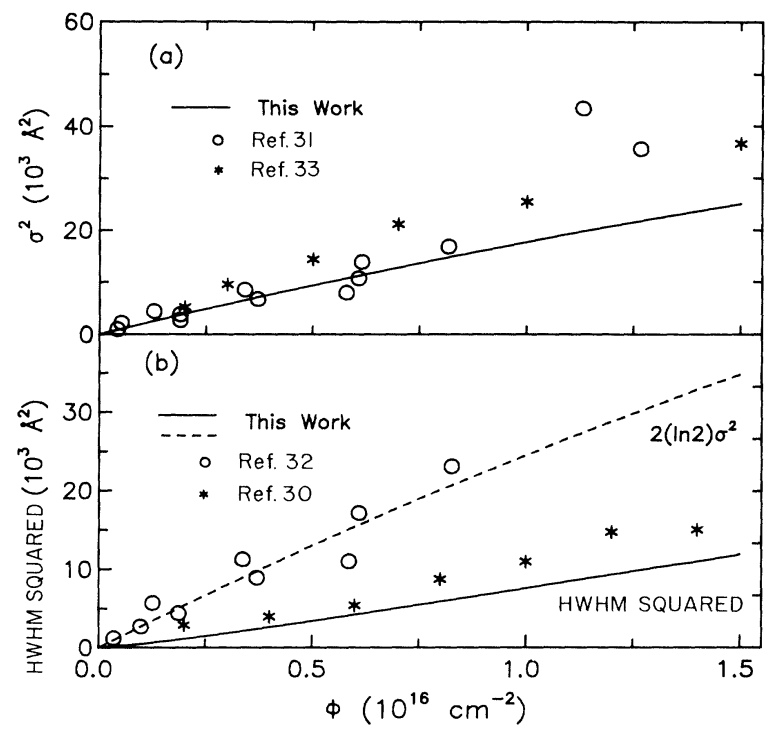

FIG. 4. Broadening vs fluence of a Pt marker in Si. For 300$\mathrm{keV} \mathrm{Xe} \rightarrow \mathrm{Si} / \mathrm{Pt} / \mathrm{Si}$.

are reported as the square of the HWHM. For the present calculations, $2(\ln 2) \sigma^{2}$ is always larger than the square of the HWHM (which has been calculated from half the full width at half maximum); this means that predicted profiles, as in Fig. 3, are not symmetric and are far from Gaussians. The experiments, on the contrary, seem to miss the lack of symmetry of the profiles, as shown in Fig. 4, where the values of the square of the HWHM calculated experimentally ${ }^{32}$ agree better with $2(\ln 2) \sigma^{2}$.

\section{CONCLUSIONS}

We have presented a powerful formalism to deal with ballistic mixing in which features such as effective atomic volume, projectile-implant effect, and surface barrier are included. This formalism has the advantage that it is, in principle, easy to drop approximations such as that of a diluted impurity, made so far in the calculations.

An important further advantage of this formalism is that it clearly spells out the models on which input quantities are based, as relocation cross sections and reflection function, in terms of input parameters like $m_{i j}, E_{d}$, and $U$.

Results obtained so far, both those reported in this work and recently, ${ }^{16-18}$ show good agreement with other theoretical calculations and reasonable agreement with experimental results. We believe that, although other mechanisms not included in our model, such as radiation enhanced diffusion and chemical forces, do play an important role in atomic mixing by ion bombardment, more experiments should be carried out paying special attention to differences between the characterization of the 
distortion of the profiles either by $\sigma^{2}$ or by the square of the HWHM.

\section{ACKNOWLEDGMENTS}

Partial funding for this work is provided by the Spanish DGICyT (Project Nos. PS88-0066 and PS89-0065). Discussions with Priv. Doz. Dr. H. M. Urbassek, and Professor G. Carter, Professor G. Falcone, and Professor R. P. Webb, were facilitated by the program of Acciones Integradas with Germany and Italy, and by the British Council. Discussions with M. M. Jakas were made possible thanks to a Sabbatical stay supported by the Spanish M.E.C.

\section{APPENDIX A: RELAXATION TO A PRESCRIBED LOCAL DENSITY}

The homogeneous relaxation model specified in Eq. (2), $\sum_{i} \alpha_{i}=1$, yields a total atomic density $\sum_{i} N_{i}$ which varies with $x$, depending on the effective atomic volume of the atoms located at $x$. One may wish to incorporate into the formalism a more general behavior of solids, which can certainly accommodate locally limited deviations from initial total density without relaxation.

Let us assume the target relaxes to a prescribed, but fluence dependent, in general, local fractional concentration

$$
\sum_{i} \alpha_{i}(x, \phi)=\sum_{i} \Omega_{i} N_{i}(x, \phi)=f(x, \phi) .
$$

If we neglect the contribution of the implanted ions, for clarity, we get

$$
\begin{aligned}
\frac{\partial f(x, \phi)}{\partial \phi}= & \sum_{i} L_{i} \alpha_{i}(x, \phi) \\
& -\frac{\partial}{\partial x}\left[f(x, \phi) \int_{0}^{x} d x^{\prime} h\left(x^{\prime}, \phi\right)\right],
\end{aligned}
$$

which, together with Eq. (1), gives a system of integrodifferential equations that determines the relaxation function $h(x, \phi)$. Equation (A2) contains a source term.

For small deviations from homogeneous relaxation, which will be the rule rather than the exception for real solids, one may write

$$
f(x, \phi) \simeq 1+\Delta(x, \phi)
$$

with

$$
\Delta(x, \phi) \ll 1 .
$$

We also expand the relaxation function $h(x, \phi)$,

$$
h(x, \phi) \simeq \sum_{i} L_{i} \alpha_{i}(x, \phi)-\xi(x, \phi),
$$

where the function $\xi(x, \phi)$ is of the same order as $\Delta(x, \phi)$, $\xi(x, \phi) \approx \Delta(x, \phi)$. Neglecting terms of second order, one gets, from Eqs. (A2) - (A5),

$$
\begin{aligned}
h(x, \phi)= & {[2-f(x, \phi)] \sum_{i} L_{i} \alpha_{i}(x, \phi)-\frac{\partial f(x, \phi)}{\partial \phi} } \\
& -\frac{\partial f(x, \phi)}{\partial x} \int_{0}^{x} d x^{\prime} \sum_{i} L_{i} \alpha_{i}\left(x^{\prime}, \phi\right),
\end{aligned}
$$

which is the explicit form of the relaxation function for a prescribed local density. For $f(x, \phi)=1$, one retrieves from Eq. (A6) the result in Eq. (3) (we are neglecting the effect of the ion). This more general formalism of the relaxation function will be discussed elsewhere ${ }^{37}$ and explicit model predictions will be reported.

\section{APPENDIX B: SERIES EXPANSION OF SOME INTEGRALS APPEARING IN THE CROSS SECTIONS}

The kind of integral that appears in Eqs. (17) and (19) is

$$
I=\int_{t_{0}}^{t_{1}} d t t^{-2 m_{i 2}}\left(t+E_{d i}\right)^{-p_{i}}
$$

In the numerical process of solving Eq. (1), this integral occurs many times so, in order to save computer time, it is evaluated by a series expansion of the form

$$
\left(t+E_{d i}\right)^{-p_{i}}=\left\{\begin{array}{l}
t^{-p_{i}} \sum_{n=0}^{\infty} K_{n}\left[\frac{E_{d i}}{t}\right]^{n}, \quad \text { if } t \geq E_{d i} \\
E_{d i}^{-p_{i}} \sum_{n=0}^{\infty} K_{n}\left(\frac{t}{E_{d i}}\right)^{n}, \quad \text { if } t \leq E_{d i},
\end{array}\right.
$$

where

$$
K_{n}=(-1)^{n} \prod_{k=1}^{n} \frac{p_{i}+k-1}{k}, K_{0}=1 .
$$

The undefined integral of Eq. (B1), once this series has been replaced in it, is 


$$
I(t)=\left\{\begin{array}{l}
I_{1}=t^{-p_{i}-2 m_{i 2}+1} \sum_{n=0}^{\infty} \frac{K_{n}}{1-n-p_{i}-2 m_{i 2}}\left(\frac{E_{d i}}{t}\right)^{n}, \quad \text { if } t \geq E_{d i}, \\
I_{2}=E_{d i}^{-p_{i}} t^{-2 m_{i 2}+1} \sum_{n=0}^{\infty} \frac{K_{n}}{1+n-2 m_{i 2}}\left(\frac{t}{E_{d i}}\right)^{n}, \quad \text { if } t \leq E_{d i} .
\end{array}\right.
$$

\section{Cascade-mixing relocation cross section}

In this case, one gets, from Eq. (17), the kind of integral in Eq. (B1) after the substitution $t=T-E_{d i}$. The limits of the integral are

$$
t_{0}=\left(\frac{|z|}{A_{i}}\right)^{1 / 2 m_{i 2}}
$$

and $t_{1}=\infty$. From Eqs. (17) and (B4) one has

$$
d \sigma_{2 i}^{\mathrm{CM}}(x, z)=d z \frac{\Gamma \mathcal{F}^{D}(x) C_{2 i}\left(1-m_{22}\right) \gamma_{2 i}^{1-2 m_{22}+m_{2 i}}}{2 N_{2} C_{22}\left(1-2 m_{22}+m_{2 i}\right) A_{i}}\left\{\begin{array}{l}
I_{1}(\infty)-I_{1}\left(t_{0}\right), \text { if } t_{0} \geq E_{d i} \\
I_{2}\left(E_{d i}\right)-I_{1}\left(E_{d i}\right)-I_{2}\left(t_{0}\right)+I_{1}(\infty), \text { if } t_{0} \leq E_{d i},
\end{array}\right.
$$

and using Eq. (B4),

$$
\begin{aligned}
d \sigma_{2 i}^{\mathrm{CM}}(x, z)= & d z \frac{\Gamma \mathcal{F}^{D}(x) C_{2 i}\left(1-m_{22}\right) \gamma_{2 i}^{1-2 m_{22}+m_{2 i}}}{2 N_{2} C_{22}\left(1-2 m_{22}+m_{2 i}\right) A_{i}} \\
& \times\left\{\begin{array}{l}
\left.\left[\frac{|z|}{A_{i}}\right)^{\left(1-p_{i}-2 m_{i 2} / 2 m_{i 2}\right.} \sum_{n=0}^{\infty} \frac{K_{n}}{n+p_{i}+2 m_{i 2}-1} \mid \frac{R_{i}\left(E_{d i}\right)}{|z|}\right]^{n / 2 m_{i 2}},|z| \geq R_{i}\left(E_{d i}\right) \\
\left.\frac{S_{i}}{E_{d i}^{p_{i}+2 m_{i 2}-1}}-E_{d i}^{-p_{i}}\left[\frac{|z|}{A_{i}}\right]^{\left(1-2 m_{i 2}\right) / 2 m_{i 2}} \sum_{n=0}^{\infty} \frac{K_{n}}{n-2 m_{i 2}+1} \mid \frac{R_{i}\left(E_{d i}\right)}{|z|}\right]^{-n / 2 m_{i 2}},|z| \leq R_{i}\left(E_{d i}\right),
\end{array}\right.
\end{aligned}
$$

where

$$
S_{i}=\sum_{n=0}^{\infty} K_{n} \frac{\left(2 n+p_{i}\right)}{\left(n+1-2 m_{i 2}\right)\left(n+p_{i}-1+2 m_{i 2}\right)} .
$$

\section{Surface-reflection function}

We start from Eq. (19) and, in order to get the integral in Eq. (B1) again, the change of variable needed is

$$
t=\left(\frac{x+x^{\prime}}{A_{i} \eta_{s}}\right)^{1 / 2 m_{i 2}}
$$

the limits of the integral become

$$
t_{0}=\left(\frac{x+x^{\prime}}{A_{i}}\right)^{1 / 2 m_{i 2}}
$$

and

$$
t_{1}=t_{0}\left(\frac{R_{i}(U)}{x}\right)^{1 / 2 m_{i 2}\left(1-4 m_{i 2}\right)} .
$$

The reflection function must vanish for

$$
\left(A_{i} U^{2 m_{i 2}}\right) / x \equiv R_{i}(U) / x \leq 1 \text {, }
$$

that is, when $x$ is greater or equal to the maximum path length traveled by one atom reflected by the surface. It is obvious then that $t_{1} \geq t_{0}$. From Eqs. (19) and (B4) one has, 


$$
W_{i}\left(x^{\prime}, x\right)=d z \frac{\Gamma \mathscr{F}^{D}\left(x^{\prime}\right) C_{2 i}\left(1-m_{22}\right) \gamma_{2 i}^{1-2 m_{22}+m_{2 i}}}{2 N_{2} C_{22}\left(1-2 m_{22}+m_{2 i}\right) A_{i}}\left\{\begin{array}{l}
I_{1}\left(t_{1}\right)-I_{1}\left(t_{0}\right), \text { if } t_{0} \geq E_{d i} \text { and } t_{1} \geq E_{d i} \\
I_{2}\left(E_{d i}\right)-I_{2}\left(t_{0}\right)+I_{1}\left(t_{1}\right)-I_{1}\left(E_{d i}\right), \quad \text { if } t_{0} \leq E_{d i} \text { and } t_{1} \geq E_{d i} \\
I_{2}\left(t_{1}\right)-I_{2}\left(t_{0}\right), \text { if } t_{1} \leq E_{d i} .
\end{array}\right.
$$

Using Eq. (B4) again,

$$
\begin{aligned}
W_{i}\left(x^{\prime}, x\right)= & d z \frac{\Gamma \mathcal{F}^{D}\left(x^{\prime}\right) C_{2 i}\left(1-m_{22}\right) \gamma_{2 i}^{1-2 m_{22}+m_{2 i}}}{2 N_{2} C_{22}\left(1-2 m_{22}+m_{2 i}\right) A_{i}} \\
& \times\left\{\begin{array}{l}
I\left(t_{1}\right)-I\left(t_{0}\right), \quad \text { if } t_{1} \leq E_{d i} \text { or } t_{0} \geq E_{d i}, \text { for } R_{i}(U) \geq x \\
\frac{S_{i}}{E_{d i}^{p_{i}+2 m_{i 2}-1}}-I_{2}\left(t_{0}\right)+I_{1}\left(t_{1}\right), \text { if } t_{1} \geq E_{d i} \text { and } t_{0} \leq E_{d i}, \text { for } R_{i}(U) \geq x \\
0, \text { for } R_{i}(U) \leq x .
\end{array}\right.
\end{aligned}
$$

${ }^{*}$ Permanent address: Departament de Física Aplicada, Universitat d'Alacant, Apt. 99, E-03080 Alacant, Spain.

${ }^{1}$ A. Gras-Marti and U. Littmark, in Vol. 155 of NATO Advanced Study Institute Series $E$, edited by R. Kelly and M. F. da Silva (Kluwer Academic, Dordrecht, 1989), p. 257.

${ }^{2}$ R. S. Averback, Nucl. Instrum. Methods B15, 675 (1986).

${ }^{3}$ W. Möller, in Vol. 155 of NATO Advanced Study Institute Series $E$, edited by R. Kelly and M. F. da Silva (Kluwer Academic, Dordrecht, 1989), p. 151.

${ }^{4}$ D. E. Harrison, Jr., C.R.C. Crit. Rev. Solid State Mat. Sci. 14, 51 (1988).

${ }^{5}$ U. Littmark and W. O. Hofer, Nucl. Instrum. Methods 168, 329 (1980).

${ }^{6} \mathrm{P}$. Sigmund and A. Gras-Marti, Nucl. Instrum. Methods 182-183, 25 (1981).

${ }^{7}$ A. Gras-Marti and P. Sigmund, Nucl. Instrum. Methods 168 , 389 (1980); 180, 211 (1981).

${ }^{8}$ R. Collins and J. J. Jiménez-Rodríguez, Radiat. Eff. Lett. 68, 19 (1982); R. Collins, J. J. Jiménez-Rodríguez, and T. Marsh, Nucl. Instrum. Methods B 8, 632 (1985); R. Collins, J. J. Jiménez-Rodríguez, M. Wadsworth, and R. Badheka, J. Appl. Phys. 64, 1120 (1988).

${ }^{9}$ P. Sigmund, A. Oliva, and G. Falcone, Nucl. Instrum. Methods 194, 541 (1982).

${ }^{10} \mathrm{G}$. Falcone and A. Oliva, Appl. Phys. A 33, 175 (1984).

${ }^{11}$ A. Oliva, R. Kelly, and G. Falcone, Surf. Sci. 166, 403 (1986).

12J. A. Peinador, I. Abril, J. J. Jiménez-Rodríguez, and A. Gras-Marti, Vacuum 39, 695 (1989).

${ }^{13}$ A. Gras-Marti, J. J. Jiménez-Rodríguez, J. Peón-Fernández, and M. Rodríguez-Vidal, Philos. Mag. 45, 191 (1982); A. Gras-Marti, J. J. Jiménez-Rodríguez, J. Peón-Fernández, M. Rodríguez-Vidal, N. P. Tognetti, G. Carter, M. J. Nobes, and D. G. Armour, Nucl. Instrum. Methods 194, 449 (1982); Vacuum 32, 433 (1982).

${ }^{14}$ U. Littmark and W. O. Hofer, Top. Curr. Phys. 37, 159 (1984).

${ }^{15}$ Top. Appl. Phys. 47, 9 (1981); 52, 11 (1983).

${ }^{16}$ J. A. Peinador, I. Abril, J. J. Jiménez-Rodríguez, and A. Gras-Marti, Nucl. Intrum. Methods B 48, 589 (1990).
${ }^{17}$ J. A. Peinador, I. Abril, J. J. Jiménez-Rodríguez, and A. Gras-Marti, Surf. Interface Anal. 15, 463 (1990).

18J. J. Jiménez-Rodríguez, I. Abril, J. A. Peinador, and A. Gras-Marti, Nucl. Instrum. Methods B 55, 681 (1991).

${ }^{19}$ P. Sigmund, Rev. Roum. Phys. 17, 969 (1972); 17, 1079 (1972).

${ }^{20}$ P. Sigmund, Phys. Rev. 184, 383 (1969); 187, 768 (1969).

${ }^{21}$ G. Falcone and P. Sigmund, Appl. Phys. 25, 307 (1981).

${ }^{22}$ M. Vicanek, J. J. Jiménez-Rodríguez, and P. Sigmund, Nucl. Instrum. Methods B 36, 124 (1989).

${ }^{23}$ F. W. Clinard, Jr. and L. W. Hobbs, in Physics of Radiation Effects in Crystals, edited by R. A. Johnson and A. N. Orlov (North-Holland, Amsterdam, 1986).

${ }^{24}$ H. H. Andersen, Appl. Phys. 18, 131 (1979).

${ }^{25}$ M. Hautala, Phys. Rev. B 41, 1759 (1990); Nucl. Instrum. Methods B 48, 504 (1990).

${ }^{26} \mathrm{~J}$. Lindhard, V. Nielsen, and M. Scharff, K. Dan. Vidensk. Selsk. Mat. Fys. Medd. 36, No. 10 (1968).

${ }^{27}$ K. B. Winterbon, P. Sigmund and J. B. Sanders, K. Dan. Vidensk. Selsk. Mat. Fys. Medd. 37, No. 14 (1970).

${ }^{28}$ I. S. Gradshteyn and I. M. Ryzhik, Tables of Integrals, Series and Products (Academic, New York, 1980).

${ }^{29}$ W. Möller and W. Eckstein, Nucl. Instrum. Methods B 7-8, 645 (1985).

${ }^{30}$ W. Möller, Nucl. Instrum. Methods B 15, 688 (1986).

${ }^{31}$ B. M. Paine, J. Appl. Phys. 53, 6828 (1982).

${ }^{32}$ B. M. Paine and M. A. Nicolet, Nucl. Instrum. Methods 209210, 173 (1983).

${ }^{33}$ A. J. Barcz, B. M. Paine, and M. A. Nicolet, Appl. Phys. Lett. 44, 45 (1984).

${ }^{34}$ H. H. Andersen and H. L. Bay, Top. Appl. Phys. 47, 145 (1981).

${ }^{35}$ B. Y. Tsaur, S. Matteson, G. Chapman, Z. Liau, and M. A. Nicolet, Appl. Phys. Lett. 35, 825 (1979).

${ }^{36}$ J. A. Peinador, Ph.D. thesis, Universidad Complutense de Madrid, 1990.

${ }^{37}$ J. A. Peinador, I. Abril, J. J. Jiménez-Rodríguez, A. GrasMarti, and G. Carter (unpublished). 